\title{
Medizinische Versorgung von Flüchtlingen: teure Hürden
}

Ein komplexes Regelwerk schränkt für derzeit 15 Monate die medizinischen Leistungen für Asylbewerber ein. Dabei deuten einige Daten deuten darauf hin, dass die Beschränkungen ein Ziel verfehlen, für das sie mit geschaffen wurden: die Kosten zu begrenzen.

„Flüchtlinge lassen sich Zähne auf Staatskosten sanieren“, mit der Zahl der Flüchtlinge gerate das „System“ in Deutschland immer häufiger an Grenzen. „Kostenexplosion in den Sozialsystemen durch Flüchtlinge.“

Nur einige Schlagzeilen bei einem kurzen Blick zum Themenkreis Medizinische Versorgung von Flüchtlingen im Internet.

Ist das wirklich so? Verschwörungstheorien sind en vogue. Soviel vorab: Dort, wo es Zahlen zu den Ausgaben für die medizinische Versorgung gibt, geben sie keinen Anlass, über Kostenexplosionen zu räso- nieren. Versuch einer Annäherung an ein komplexes Thema.

\section{Von wem ist die Rede?}

745545 Menschen stellten allein im Jahr 2016 in Deutschland einen Antrag auf Asyl. 695733 solcher Anträge entschied das zuständige Bundesamt für Migration und Flüchtlinge (BAMF) im gleichen Jahr so viele wie nie zuvor ( $\bullet$ Abb. 1).

In 256136 Fällen erkannte das Amt den Status Flüchtling oder Asylant an. In der Praxis gibt es dabei kaum Unterschiede, aber es sind 2 juristisch unterschiedliche Wege: Auch 2016 erhielt nur ein kleiner Teil der Antragsteller (0,3\%) einen Asylantenstatus nach dem vom Grundgesetzartikel 16 gewährten Recht auf politisches Asyl. Sehr viel häufiger (36,8\%) hingegen gab es eine Anerkennung nach $\S 3$ Asylgesetz als Flüchtling nach der Genfer Flüchtlingskonvention, der Deutschland 1951 beigetreten ist. Der Terminus Asyl meint im Folgenden immer beide Gruppen.

153700 Antragsteller erhielten eine vorübergehende Duldung ihres Aufenthalts. Und etwa jeder 4. Antrag, knapp 174000 , wurde abgelehnt.

Die Zahl der Asylanträge sagt nichts über die Zahl derer, die einreisen; viele Menschen stellen die Anträge erst zeitverzögert. So lag die Zahl der im easy-System registrierten neu angekommenen Menschen im Jahr 2016 bei 321371 Menschen, 2015 waren es über eine Million gewesen - die Zahl wurde später auf 890000 korrigiert, da es viele Mehrfacheinträge gab.

\section{Von welchen Kosten ist die Rede?}

Generell haben Flüchtlinge vom 1. Tag der Einreise an Ansprüche nach dem sogenannten Asylbewerberleistungsgesetz zur

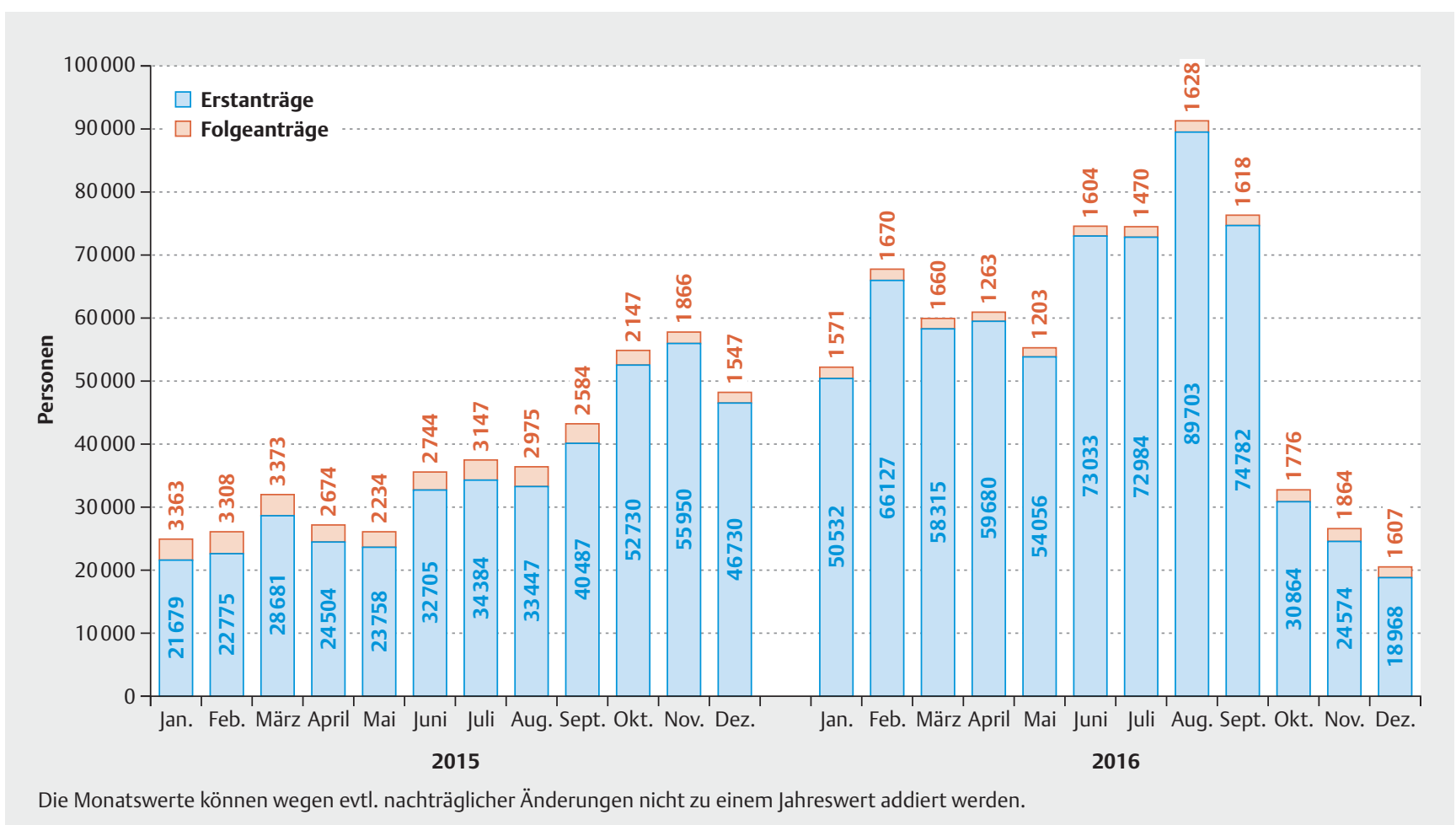

- Abb. 1 Monatliche Asylanträge seit Januar 2015. 
Sicherung des Lebensunterhalts, auf Unterkunft, Essen, Kleidung und medizinische Versorgung. Es gibt nur Schätzungen der Kosten.

- Mit gut 8000 Euro Ausgaben pro Flüchtling im Jahr nach dem sogenannten Asylbewerberleistungsgesetz kalkuliert zum Beispiel eine Studie des Finanzwissenschaftlichen Forschungsinstituts (FiFo) der Universität Köln für das Jahr 2014 [1].

- Hinzu kommen je nach Aufenthaltsdauer und Status weitere staatliche Leistungen, zum Beispiel für Sprachkurse, für die Betreuung von Kindern in Kita und Schule, oder für Integrationsmaßnahmen und Schulungen in den $\mathrm{Ar}$ beitsmarkt. Die Studie referiert ein Dutzend Hochrechnungen aus Presseartikeln namhafter Medien, die zwischen 10000 und 14000 Euro pro Jahr schätzen, Mittelwert bei 12000 Euro. Macht zwischen 10 und über 50 Milliarden jährlich für die Kassen des Staates. Laut Bundesfinanzministerium gab der Bund 2016 21,7 Milliarden Euro insgesamt für die Betreuung von Flüchtlingen aus.

- Spiegel Online bilanzierte Anfang 2016, dass der Staat am Ende im statistischen Durchschnitt an die 12000 Euro für jeden Bürger aufwendet, egal, ob er nun hier geboren wurde, oder ob er ein neu angekommener Flüchtling ist [2].

Gegen diese Kosten, auch das ist zu nennen, steht die kaum quantifizierbare Chance, dass Flüchtlinge schon bald Steuern und Sozialbeiträge zahlen könnten. Viele Experten gehen davon aus, dass das hiesige Sozialsystem in der Summe auf mittlere Sicht profitieren dürfte.

\section{Wie funktioniert die medizinische Versorgung - und wer zahlt was?}

Es ist ein ziemlich kompliziertes System an Gesetzen und Vorschriften, das mit deutscher Gründlichkeit einem Flüchtling seinen Weg in das Land weist und überaus feinziseliert auch festlegt, wann er zum Doktor darf und wann eher nicht.
Wer als Flüchtling neu nach Deutschland kommt, erhält Unterkunft zunächst nach $\S 47$ Asylgesetz maximal bis zu einem halben Jahr in einer Erstaufnahmeunterkunft eines der Bundesländer. Hier soll eine medizinische „Erstuntersuchung“ stattfinden, die wiederum nach Vorschriften des Infektionsschutzgesetzes auch dem Schutz der hiesigen Bevölkerung vor Infektionskrankheiten dienen soll. Vorschrift ist zum Beispiel ein Check auf Tuberkulose mit einer Röntgenaufnahme. Organisation und Finanzierung dieser Erstuntersuchungen und der medizinischen Versorgung in den Landeserstaufnahmestellen generell ist Sache der Bundesländer.

In Bremen zum Beispiel begann der Öffentliche Gesundheitsdienst schon 1993 mit einer eher liberalen Auslegung der Paragrafen. Der Stadtstaat sieht die Erstuntersuchung heute als niederschwelliges Angebot für medizinische Hilfe, wie Dr. Zahra Mohammadzadeh, Leiterin des Referats Migration und Gesundheit beim Gesundheitsamt Bremen und Mitarbeiterinnen letztes Jahr im Bundesgesundheitsblatt beschrieb [3]. Eine Reihenuntersuchung auf Tuberkulose findet in Bremen nicht mehr statt, aufgrund der „Fragwürdigkeit ihres medizinischen Nutzens“. Zahlen über die Kosten der medizinischen Versorgung in den Erstaufnahmeeinrichtungen bundesweit sind Mangelware.

Für die Kosten der medizinischen Versorgung aller Asylbewerber in einer langen Phase danach - bis zur Anerkennung oder Ablehnung aller Anträge - gibt es Zahlen über die Asylbewerberleistungsstatistik. Eine Gruppe um Kayvan Bozorgmehr und Oliver Razum, der eine an der Uni Heidelberg, der andere an der Uni Bielefeld, kommt auf Ausgaben von gut 7 Milliarden Euro - über einen Gesamtzeitraum von 20 Jahren, von 1994 bis 2013. Verglichen mit den Gesamtkosten des deutschen Gesundheitswesens ein „Rauschen im Hintergrund“ formuliert Kayvan Bozorgmehr (das Interview folgt in Ausgabe 3/2017).

\section{Versorgung in Kommunen}

Von der Erstaufnahme der Länder werden Flüchtlinge in Landkreise und Kommunen verteilt. Ab jetzt sind die Sozialämter vor
Ort verantwortlich für die Betreuung, einschließlich medizinischer Versorgung. Kommunen und Landkreise müssen auch die Finanzierung übernehmen, für die gesamte Dauer des Asylverfahrens gibt es damit auch keine Kosten für die Gesetzlichen Kassen. Details regelt ab jetzt das Asylbewerberleistungsgesetz, das der Bundestag 1993 verabschiedete. Ein Motiv war, Ausgaben für den Staat zu minimieren und den Zustrom von Migranten in die hiesigen Sozialsysteme zu bremsen. Damals kamen sehr viele Flüchtlinge aus den Balkankriegen nach Deutschland.

Das Mittel zum Zweck steckt vor allem in 2 Paragrafen. Paragraf 4 Asylbewerberleistungsgesetz legt fest, dass es für Asylbewerber die „erforderliche ärztliche und zahnärztliche Behandlung “ gibt, aber ganz vorrangig nur „zur Behandlung akuter Erkrankungen und Schmerzzustände“. Impfungen gibt es auch, Zahnersatz hingegen nur, wenn „unaufschiebbar“.

Darüber hinaus legt $\S 6$ fest, dass die zuständigen Behörden „sonstige Leistungen“ gewähren können, wenn sie „im Einzelfall zur Sicherung des Lebensunterhalts oder der Gesundheit unerlässlich“ sind. Das gilt momentan im Detail für:

- Asylbewerber und Flüchtlinge in den ersten 15 Monaten ihres Anerkennungsverfahrens;

- Menschen, deren Asylanträge abgelehnt wurden, die ausreisen müssen;

- Menschen, die aus humanitären Gründen geduldet werden.

Für den Besuch beim Arzt braucht ein Flüchtling jetzt vielerorts einen Behandlungsschein - abzuholen in der Regel beim zuständigen Sozialamt, oft ausgestellt für ein ganzes Quartal oder aber auch anders befristet.

Auch für Ärzte ist das, was nun zu wissen ist, durchaus komplex. Eine Kostprobe zur Verwaltungslage, in jedem Bundesland und von Ort zu Ort oft mit Variationen?

In Berlin ging es zum Beispiel bis Ende letzten Jahres um den Behandlungsschein mit der Kennzeichnung A, auch Grüner Schein genannt, abzuholen beim Landesamt für Flüchtlingsangelegenheiten. Ärzte rechnen mit diesem Schein ihre Leistungen 
mit der KV ab, der Schein selber verbleibt in der Praxis. Kostenträger ist im Behandlungsscheinverfahren in Berlin aber die AOK Nordost.

Stellt ein Doktor Verordnungen für einen Asylbewerber aus, muss er dies immer mit einem Statuskennzeichen „A“ für Asylsuchender kennzeichnen, damit dies auch für weitere Leistungserbringer wie etwa Apotheken ersichtlich ist. Bei Erwachsenen ist der Kostenträger 72802, der Kostenträgerbereich die „08“.

Weiterhin gibt es einen weißen J-Schein mit gelbem Querbalken für unbegleitete Minderjährige. Sie erhalten de facto uneingeschränkten Zugang zur Versorgung, hier muss der Arzt Kostenträger 72803 und Kostenträgerabrechnung „00“ auf die Verordnungen schreiben. Im Ärztlichen Bereitschaftsdienst wiederum muss mit einem rosa Sonderabrechnungsschein... und so weiter und so fort.

Es darf spekuliert werden, wer da wirklich den Durchblick hat. Im Zweifel hilft die Nachfrage bei KV oder Ärztekammer oder zunächst auch mal ein Blick auf das Infomaterial bei Kammer oder KVen.

In Niedersachsen gilt das gleiche Schema mit Variationen. Für Überweisungen gebe es 2 Wege; welcher gilt, vermerke der Sozialhilfeträger auf dem Behandlungsschein, erläutert die KV Niedersachsen in Hannover. Entweder muss ein Patient, der Asylbewerber ist, mit einer Überweisung erst wieder zum Sozialamt - der Arzt schreibt dann in das Feld Auftrag: „Nur zur Ausstellung eines Behandlungsscheins“. Können Ärzte gleich zu einem Kollegen überweisen, dann gehört in das Statusfeld des Personalienfelds der Hinweis „Asyl“. Damit auch der Kollege wiederum weiß, dass er nur eingeschränkte Leistungen nach $\S 4$ Asylbewerberleistungsgesetz verordnen kann. Welches Überweisungsverfahren gilt, unterscheide sich von Kommune zu Kommune - leider, kommentiert der Pressesprecher der KV Niedersachsen in Hannover, Detlef Haffke diese Vielfalt.

\section{Heikle Entscheidung über akute Erkrankung oder nicht}

Vor allem aber: Es sind die Ärzte, die in der Praxis entscheidend mit darüber befinden sollen, welche medizinischen Leistungen nun nach dem Gesetz möglich sind und welche erst mal nicht. Was also bitte ist jetzt eine akute Erkrankung, die sofort zu behandeln ist und was eine chronische, die womöglich noch etwas Zeit hat?

Chronische Erkrankungen, die ohne Behandlung zu akuten Notfällen werden, können und sollen auch nach Asylbewerberleistungsgesetz behandelt werden erklärt zum Beispiel eine „Interpretationshilfe nach Asylbewerberleistungsgesetz“, die der Freistaat Sachsen zusammen mit dortiger KV, Krankenhausgesellschaft und Ärztekammer aufgelegt hat. Außer im Notfall müsse aber immer ein Behandlungsschein vorliegen. Die Entscheidung über Ja oder Nein zu einer Gastroskopie behalten sich die Behörden im Freistaat genauso vor wie über eine „elektive“ Intervention mit dem Herzkatheter. Bei „akutem Koronarsyndrom" sollen die Patienten aber unverzüglich in eine Kardiologie mit Katheterplatz eingewiesen werden. Die Verordnung von Blutzuckermessgeräten und Teststreifen für Diabetiker erfordert hingegen wiederum eine Kostenzusage, reguläre Vorsorgeuntersuchungen seien in der Gynäkologie nicht möglich.

„Im Zweifelsfall sollten Sie immer beim Sozialamt eine Kostenübernahmeerklärung einholen“, rät auch Detlef Haffke. Es könne sonst vorkommen, dass die KV die Kosten einer Behandlung nicht übernimmt, wenn sie dafür von der zuständigen Behörde kein Geld erhalten wird.

Trotzdem scheint die Versorgung vor Ort, auch nach den komplexen Regeln, heute mancherorts zu funktionieren. Die meisten Dinge hätten sich eingespielt, meint Raimund Dehmlow, Beauftragter für Flüchtlingsfragen der Ärztekammer Niedersachsen (siehe das Interview ab S. 134). Und die Behörden würden vielerorts durchaus „großzügig“ bei Anträgen auf weitere Behandlungen agieren. Die KV Berlin erklärt sogar, dass die Senatsverwaltung für Gesundheit und Soziales in Berlin durch eine "großzügige“ Auslegung des $\S 6$ Asylbewerberleistungsgesetz de facto Asylsuchende im Leistungsumfang den GKV-Versicherten gleichstelle.

\section{Probleme mit späterer Kostenübernahme}

In Krankenhäusern ist für lange Debatten um Übernahme von Kosten vor einer Behandlung oft schon keine Zeit. Bei akuten Notfällen sollen Asylbewerber und Flüchtlinge ohne Gedanken an Behandlungsscheine zu verschwenden in die nächstbeste Klinik. Diesen Grundsatz betont auch die Deutsche Krankenhausgesellschaft (DKG). Manche Kliniken haben nach Auskunft der DKG allerdings im Nachgang von Behandlungen offenbar Mühe, ihre Kosten von den Sozialämtern erstattet zu bekommen. Nach einer hausinternen Umfrage seien die Krankenhäuser im letzten Jahr auf geschätzten 50 Millionen Euro sitzen geblieben. Abrechnungserleichterungen erhofft sich die Gesellschaft von der Einführung einer elektronischen Gesundheitskarte (eGK) auch für Asylbewerber. Sie löst in manchen Regionen mittlerweile den Behandlungsschein aus Papier ab. In Berlin etwa gibt es sie seit Anfang 2017 für alle Flüchtlinge, grüne Scheine sind dort verschwunden.

\section{Ärzte: Einschränkungen abschaffen}

Seit Langem werben Ärztevertreter dafür, die komplizierten Regeln des Asylbewerberleistungsgesetzes zu vereinfachen oder komplett zu streichen. Die Beschränkung der Behandlung auf akute Erkrankungen und Schmerzzustände sei aus „ärztlicher Sicht schlicht unethisch“, monierte Frank Ulrich Montgomery, Präsident der Bundesärztekammer im letzten Sommer in der Zeitschrift Orthopädie und Unfallchirurgie, Mitteilungen und Nachrichten. Für einen Arzt sei es nicht ausschlaggebend, warum ein Mensch nach Deutschland gekommen ist - er habe die ethische Verpflichtung, jeden Patienten gleich zu behandeln. Auch der 119. Ärztetag im Mai 2016 forderte, dass die Einschränkungen für die medizinische Versorgung von 
Flüchtlingen durch das Asylbewerberleistungsgesetz abgeschafft werden.

Daraus wurde bislang allerdings nichts. Erst im Oktober 2016 lehnte der Bundestag einen entsprechenden Antrag der Fraktion „Die Linke“ mit genau diesem Ziel ab. Ein weiterer Antrag von Bündnis 90/ Die Grünen, die psychotherapeutische und psychosoziale Versorgung zu verbessern, fiel ebenfalls durch.

\section{eGK kommt in Flächenländern oft nur schleppend voran}

Als Mindestziel fordern viele Ärzte und Wissenschaftler, allen Flüchtlingen von Anfang an statt Behandlungsscheinen gleich eine elektronische Gesundheitskarte (eGK) zu geben. Die gibt es bereits; sie sieht genauso aus wie die Gesundheitskarte eines GKV-Versicherten, außer, dass sie auf der Rückseite keinen Vermerk der Europäischen Krankenversicherung enthält und dass sie den Träger nach dem Einlesen der Daten als Asylbewerber identifiziert, dem der Arzt eben nur die eingeschränkten Leistungen nach Asylbewerberleistungsgesetz geben soll.

Auch bei der Einführung einer eGK für Flüchtlinge war das kleine Bundesland Bremen der Pionier. Der Stadtstaat gab 2005 erstmals in Deutschland die elektronische Gesundheitskarte für Asylbewerber aus. Flüchtlinge erhalten sie dort binnen 3 Monaten nach ihrer Registrierung von der AOK Bremen/Bremerhaven. Möglich macht diese Übernahme der Betreuung von Asylbewerbern durch eine Gesetzliche Kasse § 264 SGB V, nach dem Kassen auch die Versorgung Nichtversicherter übernehmen können, wenn zuständige Behörden ihnen wiederum die Kosten erstatten - nebst einem Zuschlag für ihren Verwaltungsaufwand.

Voraussetzung ist ein Rahmenvertrag zwischen einem Bundesland und einer oder mehreren Kassen, die im Auftrag die Administration und Abrechnung über die Karten leisten. Bremen schloss diesen Vertrag im Jahr 2005 mit der AOK Bremen/Bremerhaven, Hamburg folgte im Jahr 2012.
Es bleibt mit der eGK für Asylbewerber aber bei den Einschränkungen der Versorgung. Nach dem Einlesen der Karte sieht der Arzt in Bremen und Hamburg derzeit an einer Ziffer „4“ (demnächst wird es eine „9“), dass ein Asylbewerber bei ihm in der Praxis ist. „Damit weiß der Arzt, dass dieser Patient nach den Einschränkungen des Asylbewerber-Leistungsgesetzes zu behandeln ist“, erklärt Marcel Schweitzer, Pressesprecher der Hamburger Behörde für Arbeit, Soziales, Familie und Integration. Für den Arzt funktioniert die Abrechnung wie bei jedem GKV-Versicherten. Die Behandlungsdaten werden am Quartalsende zur Kassenärztlichen Vereinigung übermittelt, die KV prüft und korrigiert die Abrechnung und übermittelt die Daten an die AOK Bremen/Bremerhaven. Die wiederum erstellt eine Abrechnung für die Sozialbehörden in beiden Städten. Und bietet einige Statistiken.

So betreute die AOK Bremen/Bremerhaven zum Jahreswechsel 2015/2016 rund 35000 Flüchtlinge in beiden Stadtstaaten. Januar 2017 waren es noch 21363.

Beide Städte berichten von positiven Erfahrungen.

\section{Geringere Kosten bei einem Flüchtling verglichen mit GKV-Versichertem}

Insgesamt kostete die medizinische Versorgung der Flüchtlinge Bremen und Hamburg nach dem Asylbewerberleistungsgesetz im Jahr 2015 rund 45 Millionen Euro. Pro Kopf lagen die Ausgaben für einen Asylbewerber im Jahr zwischen Juli 2014 und Juni 2015 bei 2350 Euro. Damit koste die medizinische Versorgung von Flüchtlingen deutlich weniger als die der GKV-Patienten, berichtet Jörn Hons, Pressesprecher der AOK Bremen/Bremerhaven. Bei Letzteren liege man in der Größenordnung von 3000 Euro. Es ist eine Größenordnung, die weitere Studien bestätigen (das Interview Bozorgmehr folgt in Ausgabe 3/2017). Für Hons ist das beim Blick auf die demografischen Zahlen kein Wunder: „Über $50 \%$ der Flüchtlinge sind unter 25 Jahre - das ist eine sehr junge Gruppe.“

\section{Bessere Abrechnungs- kontrolle}

Und - Kasse wie Behörden berichten von Einspareffekten durch die eGK verglichen mit der früheren Ausgabe von Behandlungsscheinen. Hamburgs Sozialsenatorin Melanie Leonhard bezifferte Anfang 2016 die Einsparungen durch die eGK auf 1,6 Millionen Euro im 1. Jahr nach der Einführung. Das Ausstellen der Scheine entfällt, die Abrechnungen erstellt die Kasse, die dafür allerdings einen Bonus von 10 Euro monatlich je betreutem Flüchtling erhält.

Kassensprecher Hons sieht einen weiteren Kosteneinspareffekt. Mit der eGK laufe die Abrechnung der ärztlichen Leistungen nach EBM und unterliege einer Kostenkontrolle durch die GKV. Bei Versorgung über Behandlungsscheine rechneten Ärzte hingegen nach ihrer GOÄ ab. „Bei diesen Privatabrechnungen wissen wir nicht, in wie weit überhaupt geprüft wird“, erklärt Hons. Und verweist auf eine Studie der Bertelsmann Stiftung aus dem Januar 2017 zur Krankenversicherungspflicht für Beamte und Selbstständige, die deutliche Schwankungen in den Beihilfekosten für Beamte je nach Bundesland belegt, allein zwischen Bayern und Bremen um mehr als $100 \%$. „Wenn die Ärzte in Bayern bei der Privatbehandlung von Flüchtlingen per Krankenschein ähnlich großzügig abrechneten, dann dürften die Kosten dort entsprechend hoch sein“, meint auch Behördensprecher Marcel Schweitzer.

Das ist ein Stich in ein weiteres politisches und administratives Minenfeld. Denn die Bundesländer setzen die Möglichkeit zur Einführung einer elektronischen Gesundheitskarte für Flüchtlinge unterschiedlich um. Bayern, Sachsen, Sachsen-Anhalt, Mecklenburg-Vorpommern und mit dem letzten Regierungswechsel auch BadenWürttemberg wollen die Karte nicht. Berlin, Brandenburg, Niedersachsen, NRW, Schleswig-Holstein, Rheinland-Pfalz und Thüringen haben hingegen wie Bremen und Hamburg zumindest die nötigen Vereinbarungen mit Kassen geschlossen.

Nach Zahlen des GKV-Spitzenverbands wurden am 1. Mai 2016 bundesweit 
165078 Asylbewerber mit einer eGK betreut. Auch die Kassen machen sich für die eGK stark. Es sei allein schon unter Verwaltungsaspekten günstiger, den Flüchtlingen gleich die Karte zu geben, meint der Pressesprecher des GKV-Spitzenverbands in Berlin, Florian Lanz. „Diese Einsparungen überwiegen unserer Einschätzung nach auch eventuelle Mehrausgaben dafür, dass vielleicht die eine oder andere Leistung bei der eGK mehr verordnet wird als bei einem Behandlungsschein."

Dennoch stockt vielerorts die Umsetzung. Fast überall entscheiden, auch bei positivem Votum auf Landesebene, am Ende die Kommunen und Landkreise, ob sie mitmachen oder nicht. Und etliche wollen nicht, denn sie fürchten wiederum Zusatzkosten durch den Verwaltungszuschlag, den die beauftragten Kassen zusätzlich erhalten, mancherorts sind es sogar satte $8 \%$ auf die verwalteten Versorgungskosten.

Trotz einschlägiger Voten von Ärztetagen für die eGK bei Flüchtlingen bleibt das Thema offenbar auch unter Ärzten umstritten. Die Meinung zur eGK für Asylbewerber sei durchaus gespalten, berichtet Detlef Haffke von der KV Niedersachsen. Denn bei einem Behandlungsschein rechne der Arzt jede Leistung gleich auf dem Schein ab. Bei der Karte erfolgt die Abrechnung quartalsweise und hier gilt in der Regel bei Mehrfachbehandlungen in einem Quartal eine niedrigere Pauschalvergütung verglichen mit der Abrechnung über einen oder auch mehrere Behandlungsscheine.

\section{Restriktionen führen womöglich zu Mehrkosten}

Es ist, vorsichtig formuliert, nicht auszuschließen, dass die Restriktionen des Asylbewerberleistungsgesetzes die Kosten der Arztrechnung für Asylbewerber am Ende überhaupt nicht senken, sie vielmehr sogar zusätzlich nach oben treiben.

15 Monate nach der Einreise enden die Restriktionen, auch wenn ein Asylbewerber dann womöglich immer noch im Anerkennungsverfahren steckt. Jetzt erhält er nach $\S 2$ des Asylbewerberleistungsgeset- zes de facto Leistungen wie ein Hartz-IVEmpfänger mit bundesdeutscher Herkunft. Er wird Kassenmitglied und erhält eine Gesundheitskarte, die in der Praxis einen Zugang zur Regelversorgung erlaubt.

Die Kosten übernimmt vorerst weiterhin der Staat. Ebenso wie für die Gruppe der anerkannten Asylbewerber, die noch keine sozialversicherungspflichtige Stelle gefunden haben. Die Versorgung beider Gruppen erfolgt wie bei ALG-II-Empfängern bundesdeutscher Herkunft.

Die Gruppe um Oliver Razum und Kayvan Bozorgmehr legt Zahlen vor, nach denen die Versorgung von Asylbewerbern, die noch innerhalb der Frist mit einer Zugangsbegrenzung liegen, teurer kommt als bei Asylbewerbern, die nach 15 Monaten quasi Zugang zur Regelversorgung haben. „Das ist ein Effekt, den wir generell aus Gesundheitsdaten kennen“, erklärt Bozorgmehr. Eine hinausgezögerte Behandlung räche sich, wenn die Behandlung einer dann womöglich weiter fortgeschrittenen Erkrankung viel teurer werde - davon, dass dies für den Betroffenen mehr Leid bedeutet, ganz zu schweigen. Auch die Bielefelder Gesundheitswissenschaftler Oliver Razum und Judith Wenner fordern deshalb die Abschaffung der Restriktionen (das Interview folgt in Ausgabe 3/2017).

Hakeleien um die Kosten setzen sich so oder so fort. Der Staat zahlt den Kassen die Beiträge für jedes Mitglied, das mit einem ALG-II-Status geführt wird, sei es ein Asylbewerber nach 15 Monaten, ein Hartz-IVEmpfänger bundesdeutscher Herkunft oder ein anerkannter Flüchtling, der noch keine sozialversicherungspflichtige Stelle gefunden hat. Es ist ein fester Zuschuss, aktuell etwa 90 Euro im Monat. Was den Kassen aber nach deren Bekundungen nicht reicht, es gebe da eine massive „Deckungslücke“. Und sie protestieren gegen von ihnen erwartete weitere Kosten: „Aus Sicht der GKV kommt der Bund bereits bei den versicherungspflichtigen Arbeitslosengeld II Beziehenden seiner Finanzverantwortung nicht hinreichend nach; eine weitere Verschiebung von Finanzierungsverantwortung wird vor diesem Hintergrund abgelehnt“, erklärten die Juristen des GKV-Spitzenverbands in einer Stel- lungnahme im Bundestag letzten Sommer.

Ob und vor allem wie viel die Kassen wirklich drauflegen, weiß allerdings mangels valider Zahlen wieder eigentlich keiner. Bislang gab es bei der Statusmeldung auf einer eGK nur die Angabe ALG II, ohne dass erkennbar war, ob der Patient nun ein Asylbewerber ist, der länger als 15 Monate auf sein Verfahren wartet, ein anerkannter Flüchtling ist oder ein ALG-II-Empfänger bundesdeutscher Herkunft. Erst seit Anfang 2017 werde das dort differenziert, berichtet Pressesprecher Lanz vom GKVSpitzenverband. Erst das bietet jetzt eine Möglichkeit für mehr Transparenz.

So oder so erhielten die Kassen Ende 2016 schon mal vorsorglich einen Scheck in Höhe von 1 Milliarde Euro zusätzlich aus den Rücklagen des Gesundheitsfonds für, man höre und staune, „Zusatzkosten der Versorgung von Asylbewerbern“. Manch politischer Kommentar argwöhnte als Motiv eher den Wunsch der Bundesregierung, im Wahljahr 2017 womöglich generell drohende Erhöhungen von Zusatzbeiträgen für die Versicherten zu vermeiden.

Bei den Empfängern der Zuwendung gibt man sich zumindest ein wenig überrascht. Ihm sei für diese Summe keine Berechnung bekannt, die auf zugrunde liegenden Zahlen basieren würde, formuliert etwas vorsichtig GKV-Spitzenverband-Sprecher Lanz. Mithin keine Zahlen, die unterfüttern, dass die Kassen tatsächlich derartige Zusatzkosten für die Versorgung mancher Flüchtlinge haben.

Bleibt noch die Frage nach dem Zahnersatz auf Staatskosten. Der Kassenzuschuss zu Zahnersatz ist bekanntlich auch für jeden GKV-Versicherten hiesiger Herkunft schon lange gedeckelt. Es gibt maximal $70 \%$ bei ordentlich gefülltem Bonusheft - und dies wohlgemerkt für eine „Standardversorgung“. Wer da mehr will, der muss schon bislang tief in die eigene Tasche greifen. Diese Kosten seien überschaubar, meint GKV-Sprecher Lanz. Manch armer Flüchtling - und in der Tat berichten Ärzte davon, dass viele Probleme mit den Zähnen haben - wird kaum mehr erhalten als die einfachste Regelver- 
sorgung. Und das womöglich sogar aus Töpfen des Sozialsystems! Zahlen dazu? Bislang alles reine Hochrechnungen und Spekulation. Dort, wo es erste Zahlen überhaupt gibt zur medinischen Versorgung von Flüchtlingen, kann das Fazit nur lauten: Thema tiefer hängen... und möglichst rational diskutieren.

\section{Literatur}

[1] Hummel CA, Thöne M. Finanzierung der Flüchtlingspolitik. Für eine ausgewogene Finanzierung der Flüchtlingsleistungen bei Bund, Ländern und Kommunen. FiFo Berichte, Nr. 21 Februar 2016. Im Internet: http:// www.fifo-koeln.org/images/stories/fifoberichte_nr_21_fluechtlingspolitik.pdf; Stand: 13.02 .2017
[2] Diekmann F. So entstehen die Zahlen zu Flüchtlingskosten. Spiegel Online 01.03.2016. Im Internet: http://www.spiegel.de/wirtschaft/soziales/fluechtlinge-wieexperten-die-kosten-berechnen-a-1079811. html; Stand: 13.02.2017

[3] Mohammadzadeh Z, Jung F, Lelgemann M. Gesundheit für Flüchtlinge - das Bremer Modell. Bundesgesundheitsblatt Gesundheitsforschung Gesundheitsschutz 2016; 59: 561-569

Bernhard Epping 